\title{
Quantum mechanical calculation of electron spin flip in a helical undulator
}

\author{
L. N. Hand ${ }^{1}$ and A. Skuja ${ }^{2}$ \\ ${ }^{1}$ Department of Physics, Cornell University, Ithaca 14853, New York, USA \\ ${ }^{2}$ Department of Physics, University of Maryland, College Park 20742, Maryland, USA
}

(Received 24 September 2011; published 19 January 2012)

\begin{abstract}
We derive a four-component spinor wave function for an electron in a helical undulator which, in the relativistic limit, successfully reproduces all of the results of the classical calculation for the radiation angular distribution, polarization, and energy spectrum. This wave function also allows the nonclassical calculation of the spin flip. For electron energies below the several hundred GeV range, the spin-flip probability is negligible, but for higher energies and high undulator strengths it cannot be neglected if beam polarization is to be preserved, even though nonflip radiation still greatly dominates the radiation intensity. The anomalous magnetic moment $a_{e}$ is seen to play a dominant role in the helical undulator spin-flip process. The probability of spin flip is shown to have a $\gamma^{5}$ dependence on electron energy. For high energy electrons, the direction of spin flip is independent of the handedness of the undulator. As a result, at sufficiently high energy, a polarized electron or positron beam rapidly depolarizes by spontaneous radiation in the undulator. Because of the high correlation between the direction of spin flip and the handedness of the spin-flip radiation, we conjecture that it may be possible to polarize electrons by using the intense circularly polarized photons in the helical undulator to stimulate spin flip.
\end{abstract}

DOI: 10.1103/PhysRevSTAB.15.010701

PACS numbers: 41.60. $-\mathrm{m}, 29.27 . \mathrm{Hj}, 41.75 . \mathrm{Ht}$

\section{INTRODUCTION}

Relativistic electron beams passing through helical undulators are an increasingly common source of circularly polarized $x$ rays [1]. Calculations of the nature of this form of synchrotron radiation are usually done with classical electrodynamics [2]. For most purposes, it is unnecessary to take into account the wave nature of the electrons, since the classical description of the radiation process is perfectly adequate. However, in order to calculate the probability of spin flip caused by radiation, we must use a quantum mechanical calculation by solving the Dirac equation.

Historically, radiative effects on electron spin were treated for storage rings in a series of papers 30 to 40 years ago [3-5]. In a 1987 Physical Review Letters (PRL) [6,7], we showed that a simpler treatment could obtain the same results. In that paper the electron motion was described by a classical electron orbital Hamiltonian and twocomponent spinor in a Bargmann-Michel-Telegdi (BMT) Hamiltonian [8]. Both the electron orbit and the electron spin interact with both the classical magnetic guide fields and the quantized radiation field. This semiclassical approach was shown to reproduce all of the results of Sokolov and Ternov and Derbenev and Kondratenko. We calculated the damping effects on the spin, i.e., the radiation damping which leads to polarization. This approach included the effects of orbital motion on the spin and thus

Published by the American Physical Society under the terms of the Creative Commons Attribution 3.0 License. Further distribution of this work must maintain attribution to the author(s) and the published article's title, journal citation, and DOI. obtained the spin resonance conditions derived by Chao [5]. It was not necessary to solve a Dirac wave equation for the orbital motion. The phenomenological use of an anomalous moment term in the BMT equation has been vindicated by many experiments on polarization in storage rings and g-2 experiments [9].

The effect of radiation on both orbit and spin was included by treating radiation effects as a perturbation initiated by the quantized radiation field. This is standard practice (see, for example, the book by Heitler [10]). This approach derived all fluctuation and damping effects on the classical electron orbit.

Our 1987 paper [6] was for a circular storage ring. A single-pass undulator represents an entirely different physical situation, however. Radiation damping and fluctuations can be neglected. A different approach was used for the undulator calculation. We derive the fourcomponent spinor wave function for the Dirac equation including the phenomenological anomalous moment for an electron/positron in an ideal undulator field in the relativistic limit. Again, radiation is treated as a perturbation, but without explicitly using the BMT equation phenomenology. Both spin-flip and nonflip probability distributions are obtained as a result.

We introduce a form of the four-component spinor wave function which is well suited to the electron relativistic kinematic limit, $\gamma \gg 1$. In contrast with the case of circular storage rings, here we ignore stochastic radiative effects, fluctuations, and damping, since the electrons make only a single pass in the undulator.

With these assumptions, it can be shown that the spontaneous radiation in which the spin is not flipped leads to the 
same expressions for radiative power, angular distribution, and polarization as are obtained with a classical calculation. This validates the approximations we have made to obtain the wave function for the undulator.

Although spin-flip radiation is extremely rare for relativistic electron energies less than a few $\mathrm{GeV}$, in the limit of a strong undulator and $\mathrm{GeV}$ energies, it is no longer rare, although it is still infrequent compared to nonflip radiation, and thus never contributes significantly to the radiation intensity. The effect of the anomalous moment is seen to be suppressed for nonflip photon emission, but to dominate the spin-flip radiation matrix element in the relativistic limit.

The Dirac equation is solved in the high energy limit, with a phenomenological anomalous moment term added [Eq. (1) below]. As it is written, the electromagnetic fields in Eq. (1) include only the ideal classical magnetic field of the undulator.

The radiation field is included by the addition of an additional perturbative term in the Hamiltonian [Eq. (2)] containing quantized photon creation and annihilation operators in the radiation vector potential, $\vec{A}_{\text {radiation }}$. Formally, this interaction is the same as that for the classical field in Eq. (1). We can use a particular representation [11] for $\gamma^{\mu}$ and $\sigma^{\mu \nu}$ to simplify the form of the interaction with the radiation field. The coupling to the radiation field, $\vec{J}_{\text {effective, }}$, contains a piece from the Dirac current operator and a second piece from the anomalous moment operator which is proportional to $a_{e}$. Possible corrections to $\vec{J}_{\text {effective }}$ of order photon energy/electron energy have been ignored. Corrections of higher than the lowest order in the fine structure constant are ignored.

To summarize, there are two physical effects of the anomalous moment: (1) when the electron magnetic moment interacts with the classical magnetic field, possibly affecting the orbital motion (this turns out to be negligible), and (2) when the electron magnetic moment interacts with the radiation vector potential to modify photon absorption or emission.

\section{THE DIRAC EQUATION FOR THE UNDULATOR AND THE RADIATIVE PROCESS}

A fully rigorous quantum electrodynamics calculation is unnecessary in order to treat the physically observable effects of the anomalous magnetic moment. We write the Dirac equation including the anomalous moment:

$$
\left[(i \not \supset-e \not A)-m_{e}+a_{e} \frac{e}{4 m_{e}} \sigma^{\mu \nu} F_{\mu \nu}\right] \psi=0 .
$$

( $e<0$ for an electron and $e>0$ for a positron. $|e|$ is the magnitude of the electronic charge.) $a_{e}$ is the anomalous part of the electron/positron magnetic moment $[9,12]$ :

$$
a_{e} \equiv(g-2) /(2)=1159652 \ldots \times 10^{-12} .
$$

The classical undulator magnetic field is expressed in the $A_{\mu} \gamma^{\mu}=\not A$ and $F_{\mu \nu}$ terms in the Dirac equation [Eq. (1) above].

For spontaneous radiation emission we define $J_{\mu \text {,effective }} A_{\text {radiation }}^{\mu}$ as the matrix element between an initial state with zero photons and a final state with one photon.

The coupling to the electron is an effective interaction Hamiltonian:

$$
\vec{J}_{\text {effective }} \cdot \vec{A}_{\text {radiation }} \text {. }
$$

$A_{\text {radiation }}^{\mu}, F_{\text {radiation }}^{\mu \nu}$ depend on the photon polarization and the photon plane wave. We will consider only the vector components of $J_{\text {effective }}^{\mu}$, since by gauge invariance the longitudinal and time components cancel each other. The effective $\vec{J}$ for photon emission is [11]:

$$
\begin{aligned}
\overrightarrow{\mathbf{J}}_{\text {effective }} & =\overrightarrow{\mathbf{J}}_{\text {Dirac }}+\overrightarrow{\mathbf{J}}_{\text {anomalous }} \\
& =e \vec{\alpha}-a_{e} \frac{e}{2 m_{e}} \gamma^{0}(i \vec{\sigma} \times \vec{k}+\omega \vec{\sigma}) .
\end{aligned}
$$

As stated above, we obtain this form of $\vec{J}_{\text {effective }}$ by starting from the formal expression in terms of $\gamma^{\mu}$ (Dirac part) and $\sigma^{\mu \nu}$ (anomalous moment part), using a common representation for the $4 \times 4$ matrices $\gamma^{\mu}, \sigma^{\mu \nu}$ [11] and making use of the form of the solution to the Dirac equation in the undulator field, as shown in the next section.

\section{HELICAL UNDULATOR WAVE FUNCTIONS}

The explicit form of the vector potential for the static undulator magnetic field is $e \vec{A}=m_{e} c K(\cos \theta, \sin \theta, 0)$. By definition, $\theta \equiv k_{W} z, k_{W} \equiv 2 \pi / \Lambda$. $\Lambda$ is the period of the undulator. $m_{e}$ is the electron or positron mass. $K$ is the dimensionless undulator strength. In terms of the magnitude of the undulator magnetic field $B_{0}, K \equiv\left(e B_{0}\right) /\left(m_{e} c k_{W}\right)$ [1].

In our units $c$, the velocity of light, equals one. In addition, we also assume $k_{W} \equiv 1$. With this choice of units, all lengths, times, momenta, and energies are dimensionless. The subscripts $\perp$ and $\|$ refer to the $z$ axis, which is the undulator axis.

The four-component helicity solutions, in the presence of a static undulator magnetic field (transverse vector potential), are

$$
\psi=\left(\begin{array}{l}
\phi \\
\chi
\end{array}\right)
$$

The lower components can be expressed in terms of the upper components:

$$
\chi=\frac{\lambda}{E+m_{e}} \phi
$$

with $\lambda= \pm q$ and $q^{2}=E^{2}-m_{e}^{2}$. ( $q$ is the kinetic momentum, $p$ is the canonical momentum.) 
The forward moving helicity eigenstates for $\Psi$ are

$$
\Psi=\frac{1}{\sqrt{V_{0}}} e^{i(\vec{p} \cdot \vec{r}-E t)} \sqrt{\frac{E+m_{e}}{2 m_{e}}}\left(\begin{array}{c}
Z \\
\frac{\lambda}{E+m_{e}} Z
\end{array}\right) .
$$

The positive helicity solution, $\lambda=+q$, is

$$
Z_{+}=\left(\begin{array}{c}
1 \\
\frac{\beta_{+}}{2 \beta}
\end{array}\right) e^{i F}
$$

The negative helicity solution, $\lambda=-q$, is

$$
\begin{gathered}
Z_{-}=\left(\begin{array}{c}
-\frac{\beta_{-}}{2 \beta} \\
1
\end{array}\right) e^{i F} \quad \beta_{ \pm}=\beta_{x} \pm i \beta_{y} \\
F(\theta) \equiv m_{e} K \frac{p_{\perp}}{q} \sin (\theta-\alpha) .
\end{gathered}
$$

The explicit form of the velocity $\vec{\beta}$ is given below in Eq. (15). ( $p_{\perp} \ll p$ has been neglected.)

Terms of order $1 / \gamma^{4}$ have been neglected, in the spirit of the high energy limit.

The canonical transverse momentum $p_{\perp}$ is a constant of the motion in the undulator magnetic field. The canonical momentum is not equal to the kinetic momentum due to the presence of the vector potential. The transverse kinetic momentum is not constant due to the rotating nature of the magnetic field. The helicity solutions above assume that $p_{\perp}$ is small compared to the longitudinal momentum. However, we do not neglect its presence in the exponent $F$, since the change in $p_{\perp}$ when the photon is emitted is important. $p_{ \pm}$is the (complex) canonical transverse momentum of the electron.

The angle $\alpha$ is defined by the relation $p_{ \pm}=p_{\perp} e^{ \pm i \alpha}$. Note that $p_{x}=p_{\perp} \cos \alpha$ and $p_{y}=p_{\perp} \sin \alpha, q_{\perp}^{2}=p_{\perp}^{2}+$ $\left(m_{e} K\right)^{2}-2 m_{e} K p_{\perp} \cos (\theta-\alpha)$. In terms of the normalized distance along the $z$ axis, $\theta \equiv k_{W} z, q_{\|} \theta \approx$ $q \theta-\left\{\left[p_{\perp}^{2}+\left(m_{e} K\right)^{2}\right] \theta\right\} /(2 q)$. We will neglect the last term in what follows.

The effect of the anomalous moment $a_{e}$ on the wave function is to reduce the undulator strength $K$ very slightly: $K \rightarrow K\left[1-a_{e} /(2 q)\right]$. This extremely small $O[1 / \gamma]$ correction to the wave function is neglected as well as terms $O\left[1 / \gamma^{2}\right]$ and higher inverse powers of $\gamma$. Terms of order $a_{e}^{2}$ have also been neglected.

We can write the matrix elements in terms of twocomponent spinors and a two-component transition current. The two kinds of terms below correspond to the Dirac effective current, proportional to the $4 \times 4$ Dirac matrix $\vec{\alpha}$, and to the anomalous moment contribution, which is proportional to $a_{e} \gamma^{0}$ :

$$
\begin{array}{r}
\psi_{f}^{\dagger} \vec{\alpha} \psi_{i}=\left(\frac{\lambda_{f}}{E_{f}+m_{e}}+\frac{\lambda_{i}}{E_{i}+m_{e}}\right) \phi_{f}^{\dagger} \vec{\sigma} \phi_{i} \\
\psi_{f}^{\dagger} \gamma^{0} \psi_{i}=\left(1-\frac{\lambda_{f} \lambda_{i}}{\left(E_{f}+m_{e}\right)\left(E_{i}+m_{e}\right)}\right) \phi_{f}^{\dagger} \phi_{i} .
\end{array}
$$

The sign of $\lambda$ depends on the electron's helicity. Positive helicity corresponds to $\lambda=+q$, negative helicity $\lambda=-q$ ( $q$ is always positive).

The term "flip up" refers to the transition negative helicity $\rightarrow$ positive helicity, while "flip down" is the opposite transition. The photon energy is $k, E_{i}-E_{f}=k$, $q_{i}-q_{f} \approx k$. For a nonspin flip transition, the factor in Eq. (9) is $\approx 2$, while the expression Eq. (10) is very small. The anomalous moment has a negligible effect on the nonflip matrix element. In a spin-flip transition, the factor in front of $\vec{\alpha}$ will be (flip up), from Eq. (9):

$$
\frac{\lambda_{f}}{E_{f}+m_{e}}+\frac{\lambda_{i}}{E_{i}+m_{e}}=\frac{q_{f}}{E_{f}+m_{e}}-\frac{q_{i}}{E_{i}+m_{e}} .
$$

For flip up this becomes $\approx-k m_{e} / E^{2}$, assuming the photon energy is $k \ll E$.

Thus, spin flip with a Dirac moment is suppressed by a factor of $1 / \gamma^{2}$, but is not zero because the photon momentum and energy imply that $\left|q_{f}\right| \neq\left|q_{i}\right|$ and $E_{f} \neq E_{i}$.

The other term in the effective current operator [11], Eq. (10), which is proportional to $\gamma^{0}$ (and $a_{e}$ ), is not suppressed for spin flip. The presence of the anomalous moment is a radiative correction which has been experimentally verified for relativistic electrons by polarization measurements in electron storage rings. The prefactor is $\approx 2$. It is suppressed for nonflip, however.

We can express the effective current for single photon emission in the form of an operator operating only on the two-component upper part of the spinor: For flip up,

$$
\overrightarrow{\mathbf{J}}_{\text {eff }}=-e\left(\frac{k m_{e}}{E^{2}}\right) \vec{\sigma}-a_{e}\left(\frac{e}{m_{e}}\right)(i \vec{\sigma} \times \vec{k}+\omega \vec{\sigma}) .
$$

For flip down,

$$
\overrightarrow{\mathbf{J}}_{\text {eff }}=+e\left(\frac{k m_{e}}{E^{2}}\right) \vec{\sigma}-a_{e}\left(\frac{e}{m_{e}}\right)(i \vec{\sigma} \times \vec{k}+\omega \vec{\sigma}) .
$$

We have not separated the Dirac moment term for the spin flip up from the anomalous part at this point. These two parts, which can in principle interfere, have opposite signs for flip up and flip down. Also $\overrightarrow{\mathbf{J}}_{\text {eff }}(k)^{\dagger}=\mathbf{J}_{\text {eff }}(-k)$, which relates photon absorption with photon emission.

The Dirac moment term is of order $\frac{1}{\gamma^{2}}$ when compared to the anomalous moment term which is of order $a_{e}$, all other terms being comparable. This means that for $\gamma>1 / \sqrt{a_{e}}$ we can neglect the Dirac moment term when calculating the spin-flip matrix element. This condition amounts to requiring the electron energy to be greater than about $15 \mathrm{MeV}$. From now on, we will treat only the anomalous moment term in the spin-flip calculation.

\section{CALCULATION OF THE PROBABILITY}

The calculation of the probability of radiating a photon is a straightforward quantum electrodynamics calculation. 
Some of this calculation is repeated here, but $\overrightarrow{\mathbf{J}}_{\text {Dirac }}$ is replaced with the $\overrightarrow{\mathbf{J}}_{\text {eff }}$ [Eq. (3)] for spin flip.

For either the nonflip or flip cases, the probability, using box normalization, is

$$
\mathcal{P}=\frac{V_{0} m_{e}}{E} \frac{1}{(2 \pi)^{6}} \int d^{3} p^{\prime} \int d^{3} k \sum_{\text {photon polarizations }}|\mathcal{M}|^{2} .
$$

The normalization volume $V_{0}=L_{u} L^{2}$. ( $L_{u}$ is the undulator length.) The nonflip matrix element $\mathcal{M}_{\text {nonflip }}$ is

$$
\begin{aligned}
\mathcal{M}_{\text {nonflip }}= & \pm i e \sqrt{\frac{2 \pi}{k}} \int_{0}^{T_{\max }} d t \int_{0}^{L_{u}} d z \int_{-(L / 2)}^{L / 2} d x \int_{-(L / 2)}^{L / 2} d y \\
& \times e^{-i(\vec{k} \cdot \vec{r}-k t)} \Psi_{f}^{\dagger}(\vec{r}, t) \vec{\alpha} \cdot \tilde{\mathbf{e}}^{*} \Psi_{i}(\vec{r}, t) .
\end{aligned}
$$

$T_{\max }$ is the time spent in the undulator. The states $\Psi$ are given in Eqs. (6)-(8).

In taking the matrix element for this case, we obtain, to order $1 / \gamma^{2}$ :

$$
\left\langle\phi_{+}|\vec{\sigma}| \phi_{+}\right\rangle=\left(\frac{K}{\gamma} \sin \theta, \frac{K}{\gamma} \cos \theta, 1-\frac{K^{2}}{4 \gamma^{2}}\right)=\vec{\beta} .
$$

The same result is obtained for negative helicity. From the expressions for the wave function in Eqs. (6) and (7) we can write

$$
e^{i\left(F_{i}-F_{f}\right)}=e^{-i \xi \sin (\theta-\tilde{\phi})},
$$

where

$$
\xi \equiv \frac{K k_{\perp}}{\gamma}
$$

and $\tilde{\phi}$ is the azimuthal angle of the photon. We obtain this result using the definitions of $F_{i}, F_{f}$ and transverse momentum conservation

All of the standard results [1] for the harmonic content and angular distribution of the emitted photons for the nonflip case were obtained from Eq. (14) by making a harmonic analysis of $e^{i\left(F_{i}-F_{f}\right)}$. This served as confirmation that our solution [Eqs. (6)-(8)] is correct. To calculate the spin-flip matrix element, we neglect the small term proportional to $\vec{\sigma}$ (which is the dominant term for nonflip).

The matrix element for spin flip, $\mathcal{M}_{\text {flip }}$, is

$$
\begin{aligned}
\mathcal{M}_{\text {flip }}= & 2 i a_{e}\left(\frac{e}{m_{e}}\right) \sqrt{2 \pi k} \frac{1}{V_{0}} \frac{E}{2 m_{e}} \int_{0}^{T_{\max }} d t \int_{0}^{L_{u}} d z \\
& \times \int_{-(L / 2)}^{L / 2} d x \int_{-(L / 2)}^{L / 2} d y e^{-i(\vec{k} \cdot \vec{r}-k t)} \\
& \times e^{i\left[\left(\vec{p}-\vec{p}^{\prime}\right) \cdot \vec{r}-\left(E-E^{\prime}\right) t\right]} \\
& \times Z_{f}^{\dagger}(\vec{r}, t)(i \vec{\sigma} \times \hat{n}+\vec{\sigma}) \cdot \tilde{\mathbf{e}}^{*} Z_{i}(\vec{r}, t) .
\end{aligned}
$$

We can express the matrix element in terms of the explicit form of the spinors and use $\vec{S}^{ \pm}(\theta)$ to express the matrix elements of $\vec{\sigma}$ for flip up or down. In the normalization factors, we replace $\sqrt{\left(E+m_{e}\right)\left(E^{\prime}+m_{e}\right)} /\left(2 m_{e}\right)$ by $E /\left(2 m_{e}\right)$ :

$$
\begin{aligned}
\mathcal{M}_{\text {flip }}= & \mp 2 i a_{e}\left(\frac{e}{m_{e}}\right) \frac{E}{2 m_{e}} \frac{1}{V_{0}} \sqrt{2 \pi k} \int_{0}^{T_{\max }} d t \int_{0}^{L_{u}} d z \\
& \times \int_{-(L / 2)}^{L / 2} d x \int_{-(L / 2)}^{L / 2} d y e^{-i(\vec{k} \cdot \vec{r}-k t)} \\
& \times e^{i\left[\left(\vec{p}-\vec{p}^{\prime}\right) \cdot \vec{r}-\left(E-E^{\prime}\right) t\right]} e^{-i \xi \sin (\theta-\tilde{\phi})} \\
& \times\left[i \vec{S}^{ \pm}(\theta) \times \hat{n}+\vec{S}^{ \pm}(\theta)\right] \cdot \tilde{\mathbf{e}}^{*} .
\end{aligned}
$$

The integrals over space and time give rise to delta functions which express the conservation of momentum and energy. Longitudinal momentum conservation includes a contribution $e^{-i m \theta}$ from the undulator periodicity arising from the harmonic analysis of the wave function.

By definition, this harmonic analysis is

$$
e^{-i \xi \sin (\theta-\tilde{\phi})} \vec{S}^{ \pm}(\theta) \equiv \sum_{m=-\infty}^{m=+\infty} \vec{S}_{ \pm, m} e^{-i m \theta} .
$$

The Bessel functions which arise from this analysis are functions of $\xi$ :

$$
\begin{aligned}
& e^{-i \xi \sin (\theta-\tilde{\phi})} \equiv \sum_{m=-\infty}^{m=+\infty} \mathbf{a}_{\mathbf{m}} e^{-i m \theta} \quad \mathbf{a}_{\mathbf{m}}=J_{m}(\xi) e^{i m \tilde{\phi}} \\
&-\beta_{ \pm} e^{i\left(F_{i}-F_{f}\right)}=-\frac{K}{\gamma} e^{ \pm i \theta} e^{-i \xi \sin (\theta-\tilde{\phi})} \\
& \equiv \sum_{m=-\infty}^{m=+\infty} \mathbf{b}_{\mathbf{m}}^{ \pm} e^{-i m \theta} \mathbf{b}_{\mathbf{m}}^{ \pm} \\
&=-\frac{K}{\gamma} J_{(m \pm 1)}(\xi) e^{i(m \pm 1) \tilde{\phi}} .
\end{aligned}
$$

The $m$ th harmonic of the matrix element of $\vec{S}$ for flip up is

$$
\vec{S}_{+, m}=e^{i m \tilde{\phi}}\left(J_{m},-i J_{m},-\frac{K}{\gamma} e^{-i \tilde{\phi}} J_{m-1}\right) .
$$

Flip down is

$$
\vec{S}_{-, m}=e^{i m \tilde{\phi}}\left(J_{m}, i J_{m},-\frac{K}{\gamma} e^{i \tilde{\phi}} J_{m+1}\right) .
$$

Terms in the expansion with negative or zero values of $m$ are removed by the requirement of longitudinal momentum conservation when a photon is omitted: $m \geq 1$. The Bessel function arguments are $\xi \equiv K k_{\perp} / \gamma \equiv m x$, with $x$ defined by

$$
x \equiv \frac{K}{\gamma} \frac{\sin \tilde{\theta}}{1-\beta_{\|} \cos \tilde{\theta}} .
$$

$\tilde{\theta}$ is the polar angle of the emitted photon.

Flip up and flip down are no longer complex conjugates. This is a consequence of the phase factor $e^{i\left(F_{i}-F_{f}\right)}$. A spinflip asymmetry appears only in the $z$ components of $\vec{S}$. 
Because of the transverse polarization and small emission angles of the photon, it has little effect on the numerical answer.

We square the matrix element, multiply by the incident flux, and do the six-dimensional integrals, using four of them to satisfy the delta functions of energy-momentum conservation.

By our choice of units, $k_{W} \equiv 1, c \equiv 1, \beta_{z} \approx 1$. The total time in the undulator becomes a factor of $2 \pi N$.

We then have to sum over harmonics and carry out the integrals over photon angles $\tilde{\phi}, \tilde{\theta}$.

Each $k_{m}$, the photon energy for the $m$ th harmonic, contributes a factor of $m$. After the sum over photon polarizations, we have to evaluate Eq. (23) below for flip up and down separately:

$$
\sum_{m=1}^{m=\infty} m^{3}\left(\left|\vec{S}_{m}\right|^{2}-\left|\hat{n} \cdot \vec{S}_{m}\right|^{2}\right) .
$$

$\hat{n}$ is a unit vector in the direction of the emitted photon.

We know the probabilities will be dimensionless. This is not obvious in our formulas, because our assumption that $k_{W}=c=\hbar=1$ makes all energies and momenta dimensionless. In particular, $\omega_{0}=k_{W} c \equiv 1$, the undulator "frequency" has to be restored. We have to restore the dimensions at the end. For example, $1 / m_{e}$ becomes $\left(\hbar \omega_{0}\right) /\left(m_{e} c^{2}\right)$ :

$$
\begin{aligned}
k_{m} & =m \frac{\omega_{0}}{c} \frac{1}{1-\beta_{\|} \cos \tilde{\theta}} \\
\mathcal{P} & =\frac{1}{2} N \gamma a_{e}^{2} \alpha_{F S} m_{e} \int d \Omega_{k} \sum_{m}\left(\frac{k_{m}}{m_{e}}\right)^{3}\left(\left|\vec{S}_{m}\right|^{2}-\left|\vec{S}_{m} \cdot \hat{n}\right|^{2}\right) .
\end{aligned}
$$

Using azimuthal symmetry for the photon, we can do the integral over $\tilde{\phi}$ to obtain an expression for the spin-flip probability in the form of an integral over the sum of combinations of Bessel functions:

$$
\begin{aligned}
\mathcal{P}= & \pi N \gamma a_{e}^{2} \alpha_{F S}\left(\frac{\omega_{0}}{m_{e}}\right)^{2} \\
& \times \underbrace{\int_{-1}^{1} \frac{d(\cos \tilde{\theta})}{\left(1-\beta_{\|} \cos \tilde{\theta}\right)^{3}} \sum_{m} m^{3}\left(\left|\vec{S}_{m}\right|^{2}-\left|\vec{S}_{m} \cdot \hat{n}\right|^{2}\right.}_{\equiv I(K, \gamma)})
\end{aligned}
$$

The equation above defines the function $I(K, \gamma)$.

The additional factor of $\gamma$ gives for the probability of spin flip an overall $\gamma^{5}$ dependence at very high energies. The $S_{m}$ 's in terms of the Bessel functions are given below.

Flip up is

$$
\begin{aligned}
\left|\vec{S}_{m, u p}\right|^{2} & =2 J_{m}^{2}+\left(\frac{K}{\gamma}\right)^{2} J_{m-1}^{2} \\
\left|\hat{n} \cdot \vec{S}_{m, u p}\right|^{2} & =\left(J_{m} \sin \tilde{\theta}-\frac{K}{\gamma} J_{m-1} \cos \tilde{\theta}\right)^{2} .
\end{aligned}
$$

Flip down is

$$
\begin{aligned}
\left|\vec{S}_{m, \text { down }}\right|^{2} & =2 J_{m}^{2}+\left(\frac{K}{\gamma}\right)^{2} J_{m+1}^{2} \\
\left|\hat{n} \cdot \vec{S}_{m, \text { down }}\right|^{2} & =\left(J_{m} \sin \tilde{\theta}-\frac{K}{\gamma} J_{m+1} \cos \tilde{\theta}\right)^{2} .
\end{aligned}
$$

Using the fact that the photon angle is normally very small as $\beta \rightarrow 1$, we can make an approximation. In the high energy limit, as $\tilde{\theta} \rightarrow 0$,

$$
\xi=m x \quad x \rightarrow 2 K \frac{(\gamma \tilde{\theta})}{(\gamma \tilde{\theta})^{2}+1+K^{2}} \quad \text { small } \tilde{\theta} .
$$

Making the substitution $u=\gamma \tilde{\theta}$ for the photon polar angle, $x \rightarrow 2 K u /\left(u^{2}+1+K^{2}\right)$. This expression has a maximum value of $x_{\max }=K / \sqrt{1+K^{2}}$ for $u=$ $\sqrt{1+K^{2}}$. This justifies the small angle approximation, even if $K \gg 1$, since $\gamma \gg \sqrt{1+K^{2}}$ in the cases of interest, hence $x \leq 1$.

Using Eqs. (26) and (27) for spin flip up in the relativistic limit, replace $\sin \tilde{\theta}$ by $u / \gamma, u=\gamma \tilde{\theta}$ and $\cos \tilde{\theta}$ by 1 . Neglecting terms of order $1 / \gamma^{2}$,

$$
\sum_{m=1}^{\infty} m^{3}\left[\left|\vec{S}_{m}(u p)\right|^{2}-\left|\hat{n} \cdot \vec{S}_{m}(u p)\right|^{2}\right]=2 \sum_{m=1}^{\infty} m^{3} J_{m}^{2}(m x)
$$

and, for spin flip down,

$$
\begin{aligned}
& \sum_{m=1}^{\infty} m^{3}\left[\mid\left.\vec{S}_{m}(\text { down })\right|^{2}-\mid\left.\hat{n} \cdot \vec{S}_{m}(\text { down })\right|^{2}\right] \\
& \quad=\sum_{m=1}^{\infty} m^{3}\left[\left|\vec{S}_{m}(u p)\right|^{2}-\mid\left.\hat{n} \cdot \vec{S}_{m}(\text { up })\right|^{2}\right]+\mathrm{O}\left[\frac{1}{\gamma^{2}}\right] .
\end{aligned}
$$

There is no asymmetry between flip up and flip down in the limit when terms $\mathrm{O}\left[1 / \gamma^{2}\right]$ can be neglected.

The sum $2 \sum m^{3} J_{m}^{2}(m x)$ is expressed as an infinite power series in $x^{2}$ divided by $4\left(1-x^{2}\right)^{5}$ [13]. This is a special case of a Kapteyn series. A general analytic formula does not exist. Furthermore,

$$
x=x\left(\frac{K}{\gamma}, \tilde{\theta}\right) \approx x(K, u) .
$$

In the limit where Eq. (30) is a valid approximation, the $\gamma$ dependence in the integral defined in Eq. (25), $I(K, \gamma)$, is an overall factor of $\gamma^{4}$. (The important contributions to this integral come from very small photon angles, of order $1 / \gamma$.) To a very good approximation we can use $u$ as the independent variable, and write the integral in Eq. (25) as

$$
\begin{aligned}
I & \approx 8 \gamma^{4} \int_{0}^{\infty} \frac{u d u}{\left(u^{2}+1+K^{2}\right)^{3}} f(x) \\
f(x) & \equiv 2 \sum_{m=1}^{\infty} m^{3} J_{m}^{2}(m x),
\end{aligned}
$$


where $x$ is defined by Eq. (22) and its expansion in the small angle limit is given after Eq. (27). We conclude that the limiting $\gamma$ dependence of the spin-flip probability $\mathcal{P}$ is $\gamma^{5}$ overall. We define a function $\mathcal{F}(K)$ which is only a function of the undulator strength:

$$
\mathcal{F}(K) \equiv \frac{I}{\gamma^{4}}
$$

The integral for $\mathcal{F}(K)$ is calculated numerically using Eqs. (31) and (32).

With these approximations and the definition Eq. (32), $\mathcal{P}$ becomes

$$
\mathcal{P}=\pi N \gamma^{5} a_{e}^{2} \alpha_{F S}\left(\frac{\omega_{0}}{m_{e}}\right)^{2} \mathcal{F}(K)
$$

For numerical evaluation of $\mathcal{F}(K)$, we used the $\gamma \rightarrow \infty$ limit of $\left(\sin \tilde{\theta} / \gamma^{4}\right) /\left[\left(1-\beta_{\|} \cos \tilde{\theta}\right)^{3}\right] d \tilde{\theta} / d u$, which is $(16 u) /\left[\left(1+K^{2}+u^{2}\right)^{3}\right]$.

Using MATHEMATICA [14], the first 100 terms in the sum $2 \sum_{m=1}^{\infty} m^{3} J_{m}^{2}(m x)$ were added. A numerical integral over the variable $u$ in Eq. (31) from $0 \leq u \leq \infty$ was carried out to compute $\mathcal{F}(K)$.

$\mathcal{F}(K)$ is a rapidly increasing function of $K$, for example: $\mathcal{F}(1)=6.785, \quad \mathcal{F}(2)=133.6, \quad \mathcal{F}(3)=904.8, \quad \mathcal{F}(4)=$ $3652.9, \mathcal{F}(5)=10922.7$.

An excellent fit to $\ln \mathcal{F}$ vs $\ln K$ is given by the approximate numerical formula $(0.3 \leq K \leq 5)$ :

$$
\ln \mathcal{F}(K)=1.927+3.94 \ln K+0.55 \ln ^{2} K-0.094 \ln ^{3} K .
$$

\section{ENGINEERING FORMULA FOR SPIN-FLIP PROBABILITY}

The spin-flip probability from Eq. (33) is

$$
\begin{aligned}
\mathcal{P} & =N \pi a_{e}^{2} \alpha_{F S}\left(\frac{\lambda_{C}}{\Lambda}\right)^{2} \gamma^{5} \mathcal{F}(K) \\
& =5.2 \times 10^{-11} \frac{N E^{5}[\mathrm{GeV}]}{\Lambda^{2}[\mathrm{~cm}]} \mathcal{F}(K) .
\end{aligned}
$$

In Eq. (35), $N$ is the number of undulator periods, $\Lambda$ is the undulator period in $\mathrm{cm}, E[\mathrm{GeV}]$ is the electron energy in $\mathrm{GeV}$.

If the undulator strength $K=1$, and the period $\Lambda=$ $10 \mathrm{~cm}$, the spin-flip probability per undulator period for $100 \mathrm{GeV}$ electrons is $3.7 \%$. If the undulator strength is increased to $K=2$, the probability per undulator period will be $66.7 \%$. This high value of the probability means the spin will be flipped quite often in traversing an undulator with many periods.

\section{SUMMARY AND CONCLUSION}

Our solution for the wave function plus the perturbative form of the interaction with the radiation field reproduces the classical results for the characteristics of radiation from a helical undulator. The additional terms in the Dirac equation involving the anomalous moment interacting with the undulator magnetic field are negligible if the spin is not flipped. Essentially all of the radiation intensity comes from the nonflip case. Thus, the anomalous moment has no significant effect on the undulator radiation intensity even at $\mathrm{TeV}$ energies. The spin-flip process, on the other hand, is dominated by the anomalous magnetic moment. It remains rare at all energies when compared to nonflip photon emission, but the probability of spin flip by spontaneous emission is not small for sufficiently high energy and strong undulators.

The helical undulator provides a flux of circularly polarized photons from a nonflip process. This photon flux is especially high in case the helical undulator operates in an free-electron laser (FEL) mode. We conjecture that it may be possible to stimulate spin flip and to polarize the electrons in the presence of the strong photon field.

This conjecture, if supported by further calculations, could provide a practical way to polarize a high energy electron beam in an FEL. A detailed study of this effect will be presented later. This will require a realistic description of the coherent photon flux in the FEL.

\section{ACKNOWLEDGMENTS}

We would like to thank Professor Georg Hoffstaetter of Cornell for a useful and enlightening discussion. The DESY Laboratory supported the early work on polarization. A.S. acknowledges the partial support of U.S. Department of Energy. L.H. thanks the U.S. National Science Foundation for supporting his work on polarization at DESY in Hamburg, Germany. The work at DESY was a precursor to this paper.

[1] A. Hofmann, The Physics of Synchrotron Radiation (Cambridge University Press, Cambridge, England, 2004).

[2] Early undulator radiation calculations were done in the classical case by V.G. Bagrov, V.R. Khalikov, A. A. Sokolov, and I. M. Ternov, Ann. Phys. (Leipzig) 485, 1 (1973). The book by Hofmann [1] gives a complete account of the classical calculations of undulator radiation.

[3] A.A. Sokolov and I.M. Ternov, Radiation from Relativistic Electrons, American Institute of Physics Translation Series (AIP, New York, 1986).

[4] Ya. S. Derbenev and A. M. Kondratenko, Sov. Phys. JETP 35, 230 (1972). 
[5] A. W. Chao, Nucl. Instrum. Methods 180, 29 (1981); A. W. Chao and K. Yokoya, National Laboratory for High Energy Physics (KEK) Tristan Report No. 81-7, 1981 (unpublished).

[6] L. N. Hand and A. Skuja, Phys. Rev. Lett. 59, 1910 (1987).

[7] More details of the calculation of orbit-dependent spin damping effects appear in a DESY internal report: L. Hand and A. Skuja, DESY Report No. 87-126.

[8] V. Bargmann, L. Michel, and V. L. Telegdi, Phys. Rev. Lett. 2, 435 (1959).

[9] For a recent experimental and theoretical review of the status of the muon g-2 measurement, see F. Jegerlehner and A. Nyffeler, Phys. Rep. 477, 1 (2009), with 447 references. The precession in a storage ring according to the BMT equation forms the basis for extremely precise determinations of g-2.

[10] W. Heitler, The Quantum Theory of Radiation (Clarendon Press, Oxford, 1954), 3rd ed.

[11] C. Itzykson and J. Zuber, Quantum Field Theory (McGraw-Hill International Book Company, New York, 1980), Appendix A-1.

[12] The most recent experimental value for the anomalous part of the electron magnetic moment is reported by $\mathrm{D}$. Hanneke, S. Fogwell, and G. Gabrielse, Phys. Rev. Lett. 100, 120801 (2008).

[13] G. N. Watson, A Treatise on the Theory of Bessel Functions (McMillan, New York, 1944), Chap. XVII, 2nd ed.

[14] Wolfram Research, Inc., MATHEMATICA, Version 7.0, Wolfram Research, Inc., 2008. 